\title{
Application of EUVC Algorithm to Evaluate Unconnected Vehicle in A Connected Vehicle Environment
}

\author{
Hui Feng ${ }^{1, a}$, Li Lin ${ }^{\star 2, b}$ and Ting Chen ${ }^{3, c}$ \\ ${ }^{1}$ Automobile and Traffic Engineering College, Nanjing Forestry University, Nanjing, China. \\ Corresponding author. Tel.: +86 13770562578 \\ $2^{*}$ Automobile and Traffic Engineering College, Nanjing Forestry University, Nanjing, China. \\ Corresponding author. Tel.: +86 13705180025 \\ ${ }^{3}$ Automobile and Traffic Engineering College, Nanjing Forestry University, Nanjing, China. \\ Corresponding author. Tel.: +86 15295510928 \\ aemail: dongshanfenghui@126.com, *bemail: 743695641@qq.com, email: sallyct@qq.com
}

\begin{abstract}
Keywords: Connected vehicles, Vehicle situations, Adaptive traffic signal control, Simulation Abstract: Actual traffic signal control strategies rely on detectors, it detects traffic information as a control logic input. In fact, this kind of detector is based on facilities and usually called point detector which cannot directly provide the data of vehicle situation which includes speed, location, acceleration and headway etc. Meanwhile with the development of vehicles, more and more vehicles bring a series of questions, just like traffic congestion, environmental pollution and emission. Comparing with other regions This phenomenon is especially serious in intersection. With the rapid development of wireless communication technology in the emerging inter-connected vehicle system, it is possible to contact with each other between the vehicle and vehicle, vehicle and infrastructure. In this paper it proposes that connected vehicles can provide more complete statistics when closing to intersection in connected environment, at the same time, applying a new algorithm calls EUVC, when a vehicle approaches to an intersection, three sections from this area are divided to evaluate unconnected vehicles situations with different algorithms, thereby it is possible to provide complete original statistics for adaptive signal control system to improve its work efficiency and reduce a series of traffic problems. In addition, combing VISSIM with a text bed to validate whether this algorithm can work well or not.
\end{abstract}

\section{Introduction}

It has been more than 150 years since the first colored traffic was installed in Westminster city of England from 1868. In general, there are three major traffic signal control methods: fix-timed signal control, actuate signal control and adaptive traffic signal control. In order to achieve signal optimal designs, two major factor must be considered: establishing optimal objective functions and obtaining plenty of original statistics [1]. At present, adaptive traffic signal control can be described as using different sensors and testing technologies to detect real-time traffic situations and then sending original data as input values to optimal functions finally predicting the traffic trends of future short-time [2]. Current mean stream adaptive traffic signal control systems include: SCATS, SCOOT, UTOPIA and RHODES.

With the development of emerging wireless communication technology, the relations between vehicle and vehicle, vehicle and infrastructure can communicate with each other by establishing Dedicated Short Range Communications (DSRC), as we all know this technology is called as connected vehicles technology [3]. It has wide range especially in the field of traffic safety, environmental governance and Intelligent Transport System (ITS). By making full use of source, not only can it relieves traffic pressures but gets more valuable data, like: headway, location, and speed. Furthermore, comparing with old time it also helps policy maker make wise decisions. It is worth to note that if one or more connected vehicles could not communicate with each other, it just 
leads some part of market penetration rate to decline slightly and has few negative effect on whole system. In addition, comparing with installing or maintaining ring-loop or video detectors, connected vehicles system is good at saving cost.

Penetration rate plays an important role in connected vehicle, because it will influence the collection of data with the Penetration rate is variability, furthermore, if there are more connected vehicles, this system will be able to provide more real-time data, otherwise, due to the limit of data, it will be hard to predict other unconnected vehicle situations. In order to solve this question, market penetration rate should be guaranteed. Thus market penetration rate is satisfied with at least $30 \%$ in this paper. It takes at least 20 years for connected vehicles to reach $90 \%$ penetration rate.

\section{System Overview}

In order to preferably test EUVC algorithm in connected vehicle environment, a test platform is developed [4]. As it shows in figure 1, this platform consists of two models: real road environment and simulation environment.

In real road environment, connected vehicles are equipped with vehicle equipment (VE). In order to transmit basic vehicle information to road side device (RSD), this device is based on DSRC. Besides, every basic vehicle information comprised of vehicle's size, travel speed, headway, location. Basic vehicle information complies with transmission control protocol whose communication frequency is $10 \mathrm{HZ}$.

In VISSIM simulation environment, RSD consists of three parts: vehicle information database, signal controller, and signal control board. RSD transmit basic information to VISSIM simulation environment, then this system will make full use of input value for vehicle simulation unit (VSU), the results will as an output value to RSD, followed by recycling. Besides, this system will store temporary situations of every detected vehicle to calculate connected vehicles' corresponding parameters.

In general, to implement signal control strategy, signal control unites need to obtain vehicle situations from vehicle information database which will transmit connected vehicles' location and relevant parameters under DSRC. When receiving information traffic signal controller will apply this new algorithm to evaluate unconnected vehicles situations immediately.

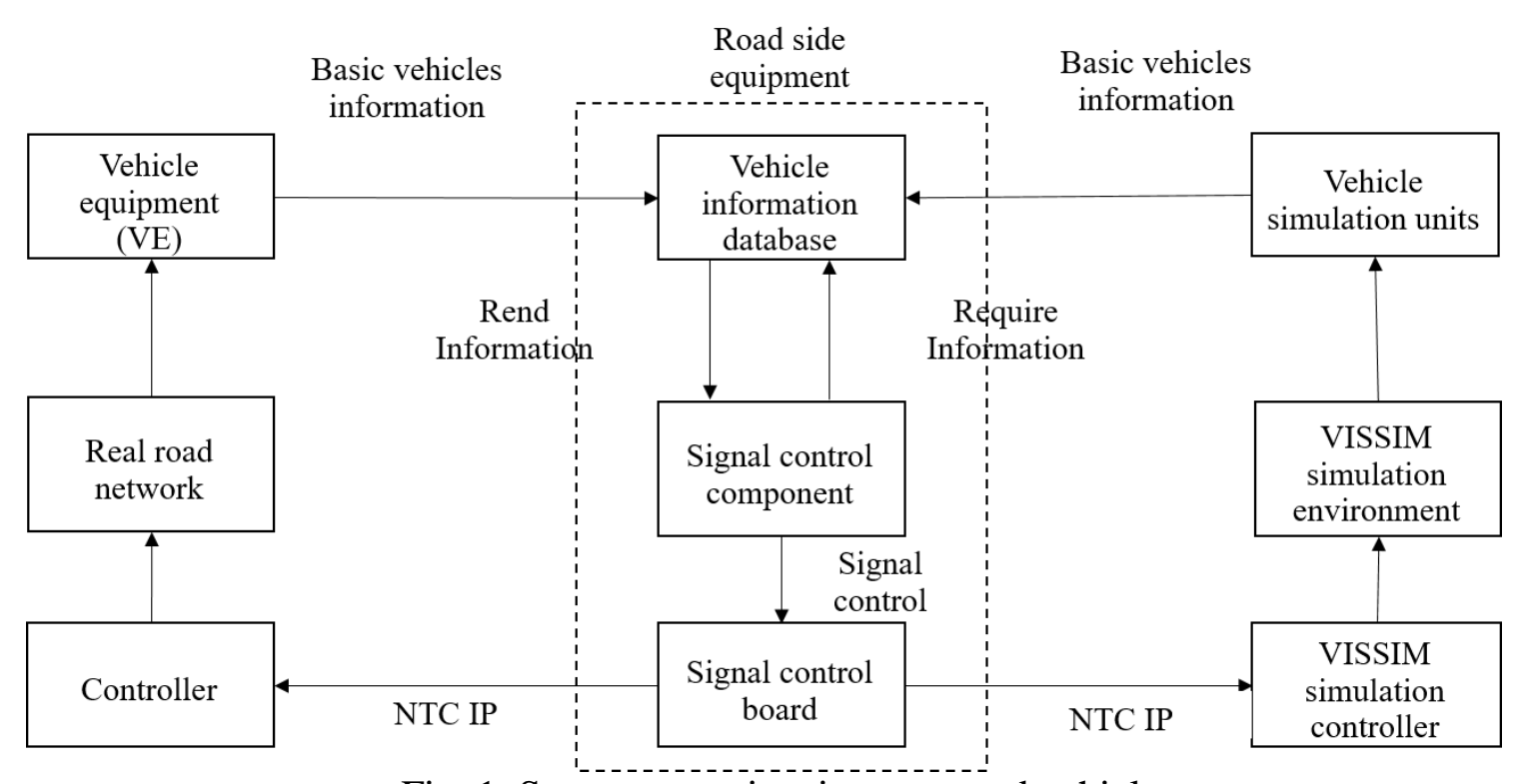

Fig. 1. System overview in connected vehicles 


\section{Evaluate Unconnected Vehicles Situations}

With the purpose of predicting unconnected vehicles driving conditions, it is necessary to obtain each vehicles information accurately on the road from connected vehicles system [5]. Here is an algorithm called EUVC (evaluate unconnected vehicles conditions-speed and location) which is responsible to deal with this question. As is shown in the figure 2 , the detail operating principle likes this:

When a car is approaching an intersection, this area is divided into three sections: a) free area, b) deceleration area, c) queuing area. Different colored rectangles represent different vehicle. The black rectangle is described as connected vehicles and the white rectangle is described as unconnected vehicles. Different algorithms adapt to their own area to estimate unconnected vehicles situations. A simple introduction of three section can be described as follows.

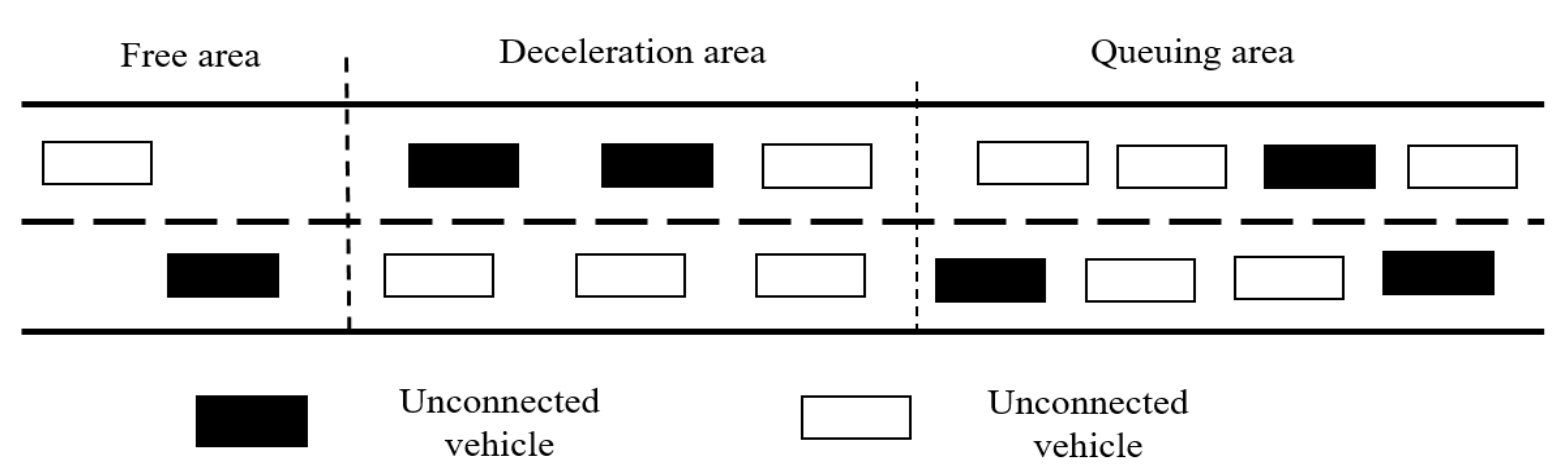

\section{Queuing area}

Fig. 2. Simple introduction of work area of EUVC

(1) Normally $\mathrm{N}=l / \mathrm{C}$, in order to estimate the number of vehicles in queuing area, it is critically important to calculate vehicle queue length. In this case, vehicle queue length is supposed as distance which always starts from stopping area to stop position, so, to find last vehicle in queuing becomes more and more important because this parameter determines queue length.

First of all, the location and braking time of last two stopping connected vehicle should be calculated and parameters are marked as: $\mathrm{D}_{1}, \mathrm{~T}_{1}, \mathrm{D}_{2}, \mathrm{~T}_{2}$

So the propagation speed between last two connected vehicles is noted as: $\mathrm{V}_{\mathrm{q}}$, like Eq. 1 .

$$
\mathrm{V}_{\mathrm{q}}=\frac{\mathrm{D}_{1}-\mathrm{D}_{2}}{\mathrm{~T}_{1}-\mathrm{T}_{2}}
$$

It should be noticed that unconnected vehicles' situations are unknown when a last connected vehicle enters the queue, besides there may some unconnected vehicles insert into queue. At this time, vehicle propagation speed in queue is consider as original speed: $\mathrm{V}_{\mathrm{q}} \cdot \mathrm{T}_{\mathrm{s}}$ is assumed as current time, by Eq. 2 to evaluate the queuing length: $l$ (real distance of last connected vehicle)

$$
l=\mathrm{D}_{1}+\mathrm{V}_{\mathrm{q}}\left(\mathrm{T}_{6}-\mathrm{T}_{1}\right)
$$

(2) Sometimes, there may be just one connected vehicle in the queue especially under low penetration rate. The Eq. 1 is not proper to measure propagation speed with the change number of connected vehicles, so Eq. 3 is established to solve this question. Assuming red-light starting time is $\mathrm{T}_{\mathrm{s}}$.

$$
\mathrm{V}_{\mathrm{q}}=\frac{\mathrm{D}_{1}}{\mathrm{~T}_{1}-\mathrm{T}_{\mathrm{s}}}
$$


Due to the last connected vehicle is used to evaluate queuing length, if average length of vehicle is $\mathrm{C}$ and $l$ is last location of vehicle, it will easy to calculate the number of vehicle in queue, $l$ is the last vehicle position.

$$
\mathrm{N}=l / \mathrm{C} \text {. }
$$

(3) When traffic demand is satisfied with random, it will lead two connected vehicles enter into queue at the same time, due to the increase of connected vehicles' number, according mention above all, the queuing length is decreasing, but propagation speed still remains the same. To avoid the mistake of Eq. 2 which will overestimate the queuing length. Thereby a new parameter will be added into Eq. 2 to offset queuing length, like: Eq. 5

$$
l=\mathrm{D}_{1}+\mathrm{V}_{\mathrm{q}} \min \left[\left(\mathrm{T}_{\mathrm{c}}-\mathrm{T}_{1}\right), \frac{\varepsilon}{\mathrm{p}}\right] .
$$

$\mathrm{P}$ is penetration rate and $\varepsilon$ is calibration parameter, $\varepsilon / \mathrm{p}$ represents adjusted value, it is obvious that the value will increase with the decrease of penetration rate. Concerning penetration rate which can be detected by detectors.

\section{Deceleration area}

According to some papers theory, since vehicles enter into deceleration area, due to drivers are influenced by the front of vehicles, they have to become rational. There is a model to describe above situation in deceleration area that divides car-following models in four sections: free-flow, following, closing and emergence. In addition, this model bases travel speed, location, acceleration on the current road network. As shown in table 1 [6], there are some parameters in four section to adjust stage of specific section.

Table 1 Parameters of car-following model

\begin{tabular}{llll}
\hline Notation & Description & Value & Unit \\
\hline$\Delta x$ & Headway & 1.96 & {$\left[\mathrm{~m} / \mathrm{s}^{2}\right]$} \\
$\Delta \mathrm{v}$ & Relative velocity & $\mathrm{V}_{\mathrm{n}}-\mathrm{V}_{\mathrm{n}-1}$ & {$[\mathrm{~m}]$} \\
$\mathrm{ABX}$ & Minimum headway at low $\Delta \mathrm{v}$ & $\mathrm{AX}+\mathrm{BX}$ & {$[\mathrm{m}]$} \\
\hline
\end{tabular}

The procedure of EUVC as shown in figure. 3. 


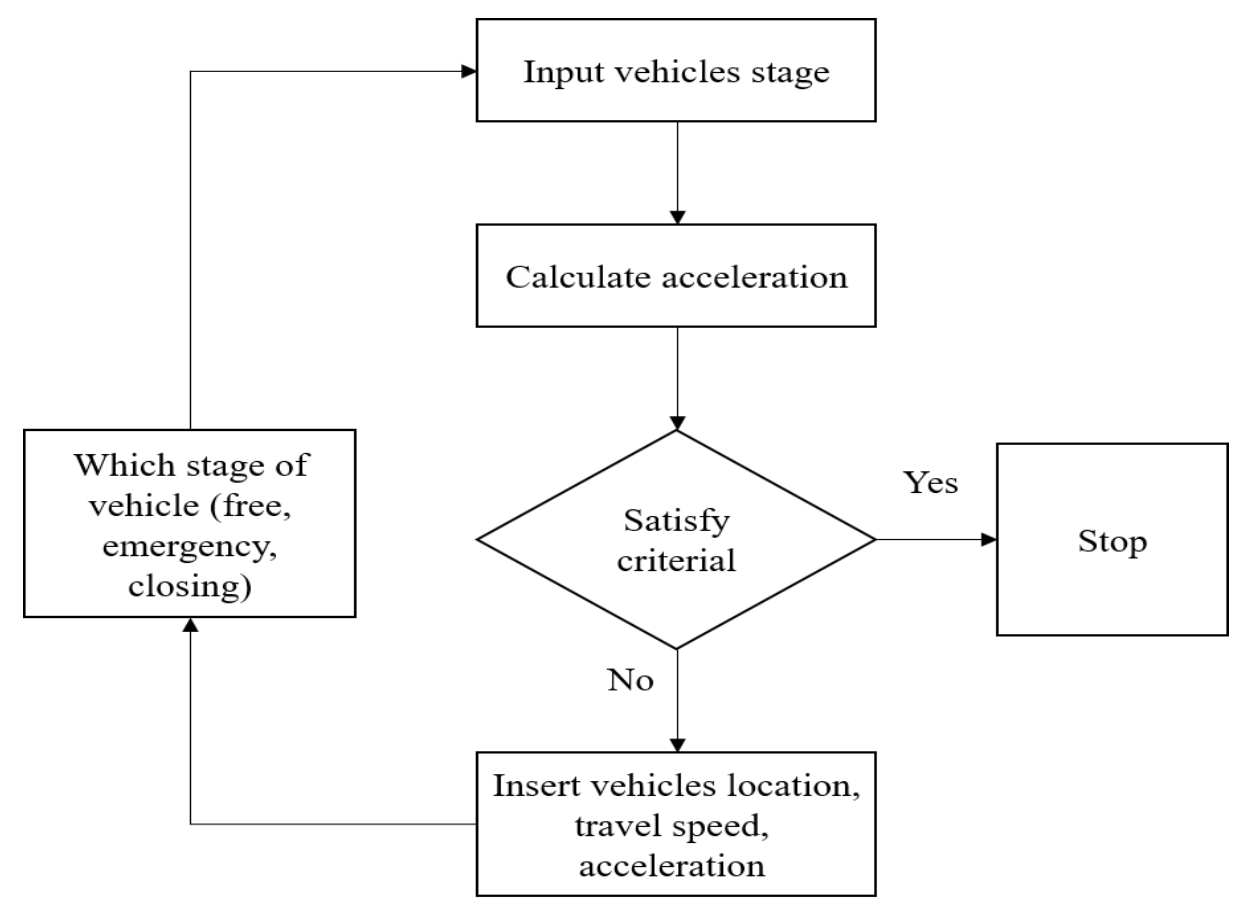

Fig. 3. Procedure of EUVC

As is shown in figure 3, it is valid to decide which kinds of vehicles belong to their own situation. Previous researches set the acceleration under actual condition instead of simulation environment. For vehicles in deceleration area, their operating states either following or closing when near to queuing area. Thus a new standard is proposed: If a vehicle is following or closing under the state and the distance between two connected vehicles more than twice the largest following distance between vehicles, it will assume that there is a vehicle to insert.

Then a series of parameters need to be calculated, including location, travel speed headway and acceleration etc. It assumes that the speed of following vehicle is $V_{n}$ and insert vehicle is $V_{n-1}$, Eq. 6 indicates that the speed of (n-1)th vehicle is determined by nth

$$
\mathrm{V}_{\mathrm{n}-1}=\mathrm{V}_{\mathrm{n}} \text {. }
$$

Due to this model has not provide the value of acceleration directly, thereby previous studies assume that the rate of acceleration change for inserting vehicles is satisfied with zero. It is worth to note that the rate of acceleration change could not indicate vehicle is stopping in deceleration area. In this paper if both leading connected vehicle (n)th and following connected vehicle (n-2)th are trying to decelerate, the insert unconnected vehicle also follows this state and slow down,. As shown in table 2 [6]. If insert vehicles cannot satisfy this criteria, the rate of acceleration change is defined as zero. Sometimes there are many factors influence the travel path of vehicle which are really difficult to predict in an exact way, hence it does not need to simulate in VISSIM. If insert vehicles stay at closing state, Eq. 7 is used to estimate headway.

$$
\Delta \mathrm{x}=\mathrm{ABX}-0.5 \frac{\left(\min \left(-\lambda_{\mathrm{a}_{\mathrm{n}}, \mathrm{v}_{\mathrm{n}}}\right)\right)^{2}}{\mathrm{a}_{\mathrm{n}}-\mathrm{a}_{\mathrm{n}-1}}
$$


Table 2 the deceleration rate between the speed of two vehicles

\begin{tabular}{ll}
\hline The speed of two vehicles & The deceleration $\left[\mathrm{m} / \mathrm{s}^{2}\right]$ \\
\hline $0-10$ & 0.91 \\
$10-20$ & 1.92 \\
$20-30$ & 1.82 \\
$30-40$ & 1.26 \\
$40-50$ & 0.67 \\
\hline
\end{tabular}

\section{Free area}

The relation between vehicle and vehicle is independent in the process of driving, applying an appropriate model to estimate unconnected vehicles is really hard in this situation. In this case, in order to obtain the number of all vehicles, connected vehicles on the road should be reassigned base on penetration rate. Besides the travel speed of unconnected vehicles are considered either restrictive speed in reality or observed speed.

\section{Conclusions}

To further evaluate EUVC algorithm for unconnected vehicles, a micro adaptive traffic signal control test bed has established which is located in Nanjing forestry university, Nanjing. In addition, combining with Part 2 to simulate in VISSIM.

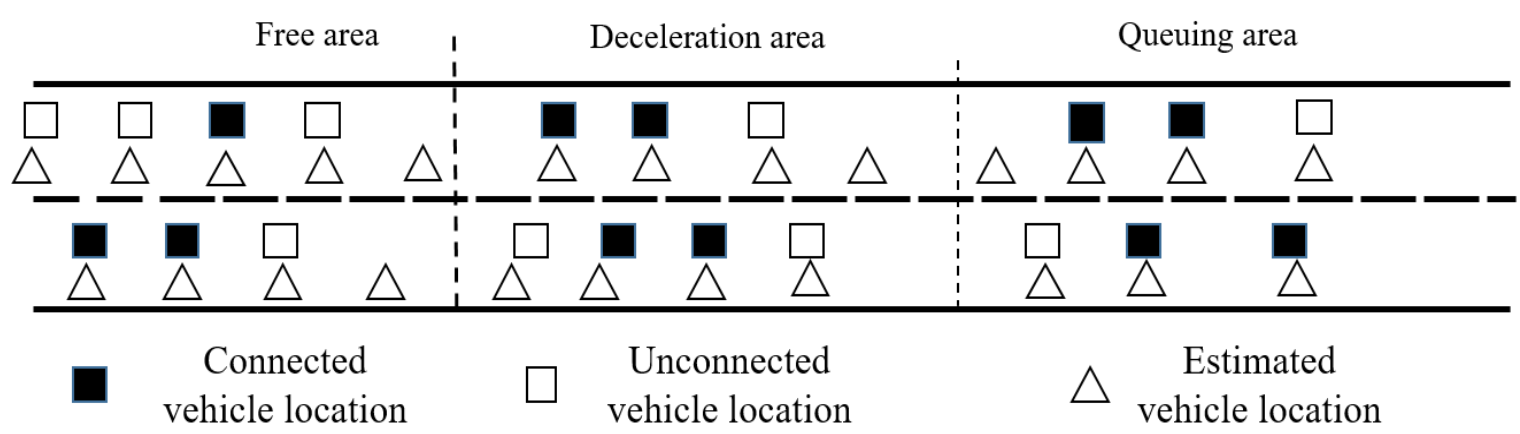

(a) the result of EUVC algorithm under $60 \%$ penetration rate in 4-phase

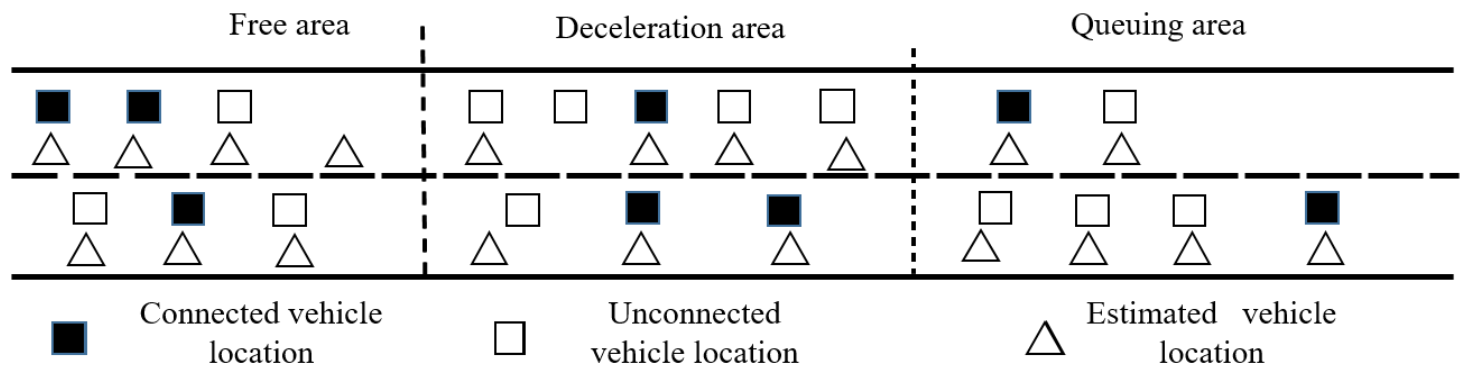

(b) the result of EUVC algorithm under $30 \%$ penetration rate in 4-phase

Fig. 4. The position of EUVC estimate under different penetration rate

As shown in figure 4, it approves that this algorithm is pretty good at estimating unconnected vehicles' location at 4-phase under different penetration rate. One of the most important reason is that EUVC can accurately estimate the propagation speed and the queuing length.

In some case, due to different driving behaviors of different divers, the distance between vehicles in real road network is larger than the distance between vehicles in deceleration area, so it is obvious that more vehicles are able to insert. In free area, vehicles stay at free state and have few negative effects on other vehicles, meanwhile, there is no adaptive model to fit this area, thereby it 
is hard to predict location. In general, the performance of this algorithm is satisfactory comparing with actual vehicles positions.

\section{Reference}

[1] Wiering, M. Multi-agent reinforcement learning for traffic light control. ICML. 2000, p. $1152-1155$

[2] Liu H, Zhang J and Cheng D. Analytical Approach to Evaluating Transit Signal Priority. Journal of transportation system enginering and information technology. Volume 8 (2008), p. 49-51

[3] Priemer, C, Friedrich, B. submitted to 12th International IEEE Conference on Intelligent Transportation Systems (2009)

[4] Jin, J, Ma, X: submitted to Proceedings of the 17th International IEEE Conference on Intelligent Transportation Systems (2014)

[5] Lee, J, Park, B. Development and evaluation of a cooperative vehicle intersection control algorithm under the connected vehicles environment. IEEE Trans. Intell. Transport. Syst. 13 (2012), p. 85-89

[6] Ma, X, Jin, J and Lei, W. Multi-criteria analysis of optimal signal plans using microscopic traffic models. Transportation Research Part D: Transport and Environment 32 (2014), p. 1-14 